\title{
The Partition Function of a Spinor Gas
}

\author{
L. F. Lemmens \\ Departement Natuurkunde, Universiteit Antwerpen RUCA, Groenenborgerlaan 171, \\ B-2020 Antwerpen, België. \\ F. Brosens, J. T. Devreese. \\ Departement Natuurkunde, Universiteit Antwerpen UIA, Universiteitsplein 1, \\ B-2610 Antwerpen, België
}

(October 21, 1999)

For a spinor gas, i.e., a mixture of identical particles with several internal degrees of freedom, we derive the partition function in terms of the FeynmanKac functionals of polarized components. As an example we study a spin-1 Bose gas with the spins subjected to an external magnetic field and confined by a parabolic potential. From the analysis of the free energy for a finite number of particles, we find that the specific heat of this ideal spinor gas as a function of temperature has two maxima: one is related to a Schottky anomaly, due to the lifting of the spin degeneracy by the external field, the other maximum is the signature of Bose-Einstein condensation.

05.30.Jp, 03.75.Fi, 32.80.Pj

\section{INTRODUCTION}

In this paper we extend our approach, introduced in [1,2] for a class of interacting polarized quantum systems, to systems with internal degrees of freedom. After a review and a generalization of our methods to the unpolarized case, their feasibility is tested on a relatively simple confined model system of spin-1 bosons in an external magnetic field. The partition function, the specific heat and the susceptibility of this trapped spinor gas are evaluated for 100 and 1000 particles. Explicit analytical results in closed form were derived for 6 particles using symbolic algebra. 
In the thermodynamic limit $(N \rightarrow \infty$ with a fixed density $\rho=N / V)$, Ginibre [3] provided a Feynman-Kac functional description of the quantum statistical equilibrium for systems with internal degrees of freedom, based on the grand canonical ensemble [4]. This approach, applied to quantum systems [5],6] and quantum plasma's [0],8], has recently been generalized by Cornu [9] to mixtures of identical particles in an external magnetic field. A review of the methodology can be found in [10] with emphasis on low-density Coulomb systems.

The approach which we present here is based on a different description [11,12], although both Ginibre's and our approach have in common the replacement of second quantization by a direct use of the permutation symmetry in a path integral. But there are essential differences in the way that the finite number of particles is incorporated in the theory. Furthermore, we treat here an unpolarized mixture of identical particles.

Our approach avoids the thermodynamical limit and keeps the relations between the density of states, the partition function for $N$ particles and their generating function mathematically exact [13]. These statistical quantities are shown to be transforms of each other, i.e., they are not separately defined on the basis of properties of an ensemble. The partition function $Z_{N}(\beta)$ is the Laplace transform of the distribution of states $\Omega_{N}(E)$ with $\beta$ adjoint to the energy $E$. The generating function $\Xi(\beta, \gamma)$ of the partition function is a Z-transform of $Z_{N}(\beta)$ with the fugacity $\gamma$ adjoint to the number of particles. For a harmonic model with interactions [2,13] we could show, using the appropriate saddle-point methods reliable for the singular structure of the generating function at large $N$, that these transforms give the well known relation between the fugacity -and thereby the chemical potential- and the number of particles. Our derivation was largely inspired by the inversion method used by Fowler and Darwin [12] to obtain the relation between the temperature and the internal energy. The temperature as a measure for the internal energy and the chemical potential as a characterization of the number of particles are quantities obtained from the theory, whereas in an ensemble-based approach (valid in the thermodynamic limit), their definitions belong to the theory. A similar statistical methodology has been recently proposed by Bormann et al. 
114 as a new approach for systems with a finite number of particles, although their method is only applicable for a partition function of a polarized system without any particle-particle interaction.

Some models of harmonically confined systems with harmonic two-body interactions can be solved exactly. They have been studied using other techniques by many authors [15]19]. The classical version of the model was already studied by Newton [20,21]. The reason for the exact solvability is the reduction to a center-of-mass evolution and an evolution of the other degrees of freedom relative to the center of mass, the latter being independent of each other [22]. Despite the conceptual simplicity of this approach, the actual calculation turns out to be quite involved. Indeed, a general two-body interaction requires a cumulant expansion of the exponential in the Feynman-Kac functional with the implication that $n$-point correlation functions have to be calculated for each higher order cumulant. These point correlation functions have their own generating functions, which have to be inverted separately. A loop, as is well known from a field theoretic approach to the many-body problem, manifests itself in the irreducible part of a $n$-point correlation function. In principle, these loops can be used to simulate a many-body system [5] or are used in a Mayer cluster expansion [7,23] of the statistical quantities relevant for stability studies of low-density Coulomb systems. Although this prescription is general, we have applied it only to the first order cumulant [24], in using the Jensen-Feynman variational principle to optimize the free energy for realistic interaction potentials. The second cumulant would already require a four-point correlation function.

Another extension of Ginibre's approach [5,9], considered in this paper, concerns the spin degrees of freedom. We have to project the full $N$-body propagator for distinguishable particles on the irreducible representations of the permutation group. In the calculation of the partition function of the model we only need the diagonal part with respect to the spin degrees of freedom. This means that only those propagations have to be considered where particles start and end in the same spin state after some Euclidean time lapse. This simplification cannot be made in the calculation of a $n$-point correlation function. In that case the evolution of a spin state should be described by a continuous-time Markov process 
with discrete states [25]. These states serve as indices on the sample paths in the FeynmanKac functional. Also when charges are present, the influence of the magnetic field on the charged particles can be taken into account with the same methods [26].

The care given to the $N$-body aspects in our formulation originates from the fact that a crossover from a density dependent behavior to a behavior dependent on the number of particles is experimentally accessible. It has become possible to produce assemblies of atoms whose internal energy is so small, that quantum effects such as Bose-Einstein condensation or Fermi-Dirac degeneracy become observable [27 30]. The theoretical challenge posed by these systems is their particular kind of confinement and the relatively low number of atoms involved. This low number of atoms suggests that there is a regime where the number of particles is more important than their density. The study of such a crossover excludes the thermodynamical limit as an investigation tool [31]. The energy levels are primarily determined by the trapping potential, rather than by the confining volume used in most quantum-statistical studies. In the earliest experimental realizations of Bose-Einstein condensation the system was restricted to a specific internal degree of freedom; in present day studies this condition has been relaxed leading to the so-called trapped spinor quantum gases [32 34], where an equilibrium over the internal degrees of freedom can be reached.

In the present paper we illustrate the calculation techniques for a boson gas with three internal degrees of freedom. A preliminary report on the results of this investigation can be found in [35. The paper is organized as follows. In section II we derive the quantum mechanical partition function of a mixture of identical particles, given only the total number of particles. In order to achieve this goal, we go through a number of steps: first we derive a partition function conditioned on a particular state of the internal degrees of freedom and a given number of particles as a Feynman-Kac functional 36 38 that can be obtained explicitly by path integral calculations, at least for the model under consideration. The resulting propagator is projected on the symmetric or antisymmetric irreducible representation of the permutation group according to the boson or the fermion character of the particles. The cyclic decomposition of the permutations imposes a constraint on the summations over the 
cycle lengths. This difficulty is circumvented by introducing a generating function for each conditioned partition function, which can be inverted. The partition function of the mixture is then obtained as an appropriate combination of the conditioned partition functions, from which the free energy and the other thermodynamical quantities of the mixture result. We describe these steps using an harmonic boson model, supplied with an homogeneous external magnetic field, i.e., with a Zeeman splitting of the spin degrees of freedom. In section III the free energy, the susceptibility and the specific heat will be obtained with emphasis on the low temperature properties and the thermal fluctuations of a boson gas. Finally, in section IV we discuss the method and the obtained results, and we conclude the paper with a brief summary of the work.

\section{QUANTUM STATISTICS OF MIXTURES}

In this section we provide the necessary background material to formulate a quantum statistical theory of mixtures of a fixed number of $N$ identical particles with internal degrees

of freedom. Using the same approach as we developed [1,2] for particles without internal degrees of freedom, we have to describe the state space and the evolution on that state space using a process and a Feynman-Kac functional averaged over that process. We also have to indicate how the projection on the irreducible representations of the permutation group has to be done in order to ensure the indistinguishability of those particles that are in the same state of their internal degrees of freedom. Finally, we give an expression for the partition function of the mixture.

\section{A. The propagator of the harmonically confined model}

The quantum model which we consider contains $N$ identical particles kept together in a confining potential given by

$$
V_{1}=\frac{1}{2} \sum_{m=-s}^{s} \Omega_{m}^{2} \sum_{k=1}^{N_{m}} \mathbf{r}_{m, k}^{2}
$$


where $\mathbf{r}_{m, k}$ is the position vector of the $k^{\text {th }}$ particle in state $m$. (Natural units with $\hbar$ and the particle mass equal to unity are used througout this paper.) The frequencies $\Omega_{m}$ are related to the curvature of the parabolic confinement potential, which in principle might be different for each internal degree of freedom. We assume that there are $N_{m}$ particles that occupy the state $m$. The total number of particles is obtained from

$$
N=\sum_{m=-s}^{s} N_{m}
$$

In the absence of interparticle interactions and for distinguishable particles, the propagator for the spatial degrees of freedom can be written as a product

$$
K_{D}\left(\left\{\mathbf{r}_{m, k}^{\prime \prime}\right\}, \beta \mid\left\{\mathbf{r}_{m, k}^{\prime}\right\}, 0\right)=\left.\prod_{m=-s}^{s} \prod_{k=1}^{N_{m}} K\left(\mathbf{r}_{m, k}^{\prime \prime}, \beta \mid \mathbf{r}_{m, k}^{\prime}, 0\right)\right|_{\Omega_{m}},
$$

where $\left\{\mathbf{r}_{m, k}^{\prime}\right\}$ represents a configuration of all the particles in the internal state $m$, and with

$$
\left.K\left(\mathbf{r}_{a}, \beta \mid \mathbf{r}_{b}, 0\right)\right|_{w}=\left(\frac{w}{2 \pi \sinh w \beta}\right)^{\frac{3}{2}} \exp \left[-\frac{w}{2} \frac{\left(\mathbf{r}_{a}^{2}+\mathbf{r}_{b}^{2}\right) \cosh w \beta-2 \mathbf{r}_{a} \cdot \mathbf{r}_{b}}{\sinh w \beta}\right] .
$$

We assume that the internal degrees of freedom behave like a spin in an external homogeneous magnetic field $\mathbf{B}$, described by the Hamiltonian

$$
\mathcal{H}=-\mu \mathbf{B} \cdot \mathbf{S} .
$$

Introducing for each particle the states characterized by the value $m$ of the spin component along the quantization axis

$$
S_{z}|s, m\rangle=m|s, m\rangle, \quad m=-s,-s+1, \ldots, s,
$$

one finds that the Euclidean time evolution for the spin states of the particle is given by:

$$
\mathcal{K}_{s}\left(m^{\prime \prime}, \beta \mid m^{\prime}, 0\right)=\left\langle s, m^{\prime \prime}|\exp -\beta \mathcal{H}| s, m^{\prime}\right\rangle .
$$

This propagator can be written in the spectral representation by introducing a unitary matrix $U$ that diagonalizes $\mathcal{H}$,

$$
U \mathcal{H} U^{-1}=-\mu|\mathbf{B}| S_{z}
$$


as follows

$$
\mathcal{K}_{s}\left(m^{\prime \prime}, \beta \mid m^{\prime}, 0\right)=\sum_{m=-s}^{s}\left\langle s, m^{\prime \prime}\left|U^{-1}\right| s, m\right\rangle e^{\beta \omega_{m}}\left\langle s, m|U| s, m^{\prime}\right\rangle,
$$

with $\omega_{m}=\mu m|\mathbf{B}|$.

Disregarding the statistics for the time being, the propagator for all the (distinguishable) particles including the internal degrees of freedom thus becomes:

$$
K_{D}\left(\left\{\mathbf{r}_{m, k}^{\prime \prime}\right\}, \beta \mid\left\{\mathbf{r}_{m, k}^{\prime}\right\}, 0\right)=\prod_{m=-s}^{s}\left[\left.e^{N_{m} \beta \omega_{m}} \prod_{k=1}^{N_{m}} K\left(\mathbf{r}_{m, k}^{\prime \prime}, \beta \mid \mathbf{r}_{m, k}^{\prime}, 0\right)\right|_{\Omega_{m}}\right] .
$$

The quantum evolution of the system is now defined as follows: the particles are confined by a harmonic potential that may differ according to their spin state $m$, and the state $m$ of the $j^{\text {th }}$ particle evolves as a spin- $m$ state in a magnetic field. This model is an idealization because interaction between the spins of different particles is left out, spin-orbit coupling or pair formation is not considered, and there is no spatial two-body interaction. But in the assumption that these interactions can be included, this simplified model still has to be studied as a zero order approximation.

\section{B. The permutation symmetry and the generating function}

From the propagator $K_{D}$ for the distinguishable particles, the propagator $K_{\text {mixt }}$ of the

mixture, taking into account the fermion or boson statistics, can be obtained by using the antisymmetric or symmetric projection as documented in [11, 12]:

$$
K_{\text {mixt }}\left(\left\{\mathbf{r}_{m, k}^{\prime \prime}\right\}, \beta \mid\left\{\mathbf{r}_{m, k}^{\prime}\right\}, 0\right)=\prod_{m=-s}^{s} e^{N_{m} \beta \omega_{m}} \mathbb{K}_{I}\left(\left\{\mathbf{r}_{m, k}^{\prime \prime}\right\}, \beta \mid\left\{\mathbf{r}_{m, k}^{\prime}\right\}, 0\right)
$$

The expression for the propagator $\mathbb{K}_{I}\left(\left\{\mathbf{r}_{m, k}^{\prime \prime}\right\}, \beta \mid\left\{\mathbf{r}_{m, k}^{\prime}\right\}, 0\right)$ of identical particles in the same spin state $m$ is

$$
\mathbb{K}_{I}\left(\left\{\mathbf{r}_{m, k}^{\prime \prime}\right\}, \beta \mid\left\{\mathbf{r}_{m, k}^{\prime}\right\}, 0\right)=\left.\frac{1}{N_{m} !} \sum_{P} \xi^{P} \prod_{k=1}^{N_{m}} K\left(\mathbf{r}_{m, P(k)}^{\prime \prime}, \beta \mid \mathbf{r}_{m, k}^{\prime}, 0\right)\right|_{\Omega_{m}},
$$


which represents a Feynman-Kac functional of the polarized components with spin state $m$, including all their permutations $P$. Knowing the propagator, the partition function for a given distribution $N_{-s}, \ldots, N_{s}$ can be expressed as a multiple integral:

$$
Z\left(\beta \mid N_{-s}, \ldots, N_{s}\right)=\left[\prod_{m=-s}^{s} \prod_{k=1}^{N_{m}} \int d \mathbf{r}_{m, k}\right] \prod_{m=-s}^{s} e^{N_{m} \beta \omega_{m}} \mathbb{K}_{I}\left(\left\{\mathbf{r}_{m, k}\right\}, \beta \mid\left\{\mathbf{r}_{m, k}\right\}, 0\right) .
$$

The calculation for each spin degree of freedom then proceeds in complete analogy with the polarized case [1], leading to a partition function of a mixture with a given composition of internal degrees of freedom

$$
Z\left(\beta \mid N_{-s}, \ldots, N_{s}\right)=\prod_{m=-s}^{s} e^{N_{m} \beta \omega_{m}} \mathbb{Z}\left(\beta \mid N_{m}\right)
$$

where $\mathbb{Z}\left(\beta \mid N_{m}\right)$ is the partition function of $N_{m}$ spin-polarized particles:

$$
\mathbb{Z}\left(\beta \mid N_{m}\right)=\left[\prod_{k=1}^{N_{m}} \int d \mathbf{r}_{m, k}\right] \mathbb{K}_{I}\left(\left\{\mathbf{r}_{m, k}\right\}, \beta \mid\left\{\mathbf{r}_{m, k}\right\}, 0\right)
$$

Of course, if the total number $N$ of particles is fixed, all combinations of the $N_{-s}, \ldots, N_{s}$ (with $\sum_{m=-s}^{s} N_{m}=N$ ) are possible and we obtain the following expression for the partition function of the mixture:

$$
\begin{gathered}
\mathfrak{Z}_{\text {mixt }}(\beta \mid N)=\sum_{N_{-s}=0}^{N} \sum_{N_{-s+1}=0}^{N} \ldots \sum_{N_{s}=0}^{N} C\left(N_{-s}, \ldots, N_{s}\right) Z\left(\beta \mid N_{-s}, \ldots, N_{s}\right), \\
C\left(N_{-s}, \ldots, N_{s}\right)=\frac{N ! \Theta\left(N=\sum_{m=-s}^{s} N_{m}\right)}{N_{-s} ! N_{-s+1} ! \ldots N_{s} !}
\end{gathered}
$$

where $C\left(N_{-s}, \ldots, N_{s}\right)$ is a multinomial coefficient in which $\Theta\left(N=\sum_{m=-s}^{s} N_{m}\right)$ expresses the constraint on the summations, i.e., $\Theta(x)=1$ if the logical variable $x$ is true, and $\Theta(x)=0$ if the logical variable $x$ is false. Using the expression for the joint partition function $\mathfrak{Z}_{\text {mixt }}(\beta \mid N)$ in terms of the polarized partition functions $\mathbb{Z}\left(\beta \mid N_{m}\right)$ one readily obtains:

$$
\mathfrak{Z}_{\text {mixt }}(\beta \mid N)=\sum_{N_{-s}=0}^{N} \sum_{N_{-s+1}=0}^{N} \ldots \sum_{N_{s}=0}^{N}\left[C\left(N_{-s}, \ldots, N_{s}\right) \prod_{m=-s}^{s} \mathbb{Z}\left(\beta \mid N_{m}\right) e^{N_{m} \beta \omega_{m}}\right] .
$$

This is the partition function that will be analyzed in the next section. We consider there a gas of atoms with Bose-Einstein statistics confined in a harmonic potential, without 
interparticle interactions, and with the ability to adjust their internal degrees of freedom in order to attain thermodynamical equilibrium. If the system is prepared in such a way that the total $z$ component of all the spins can be fixed, then this constraint can be taken into account using a technique developed in [39]. In this case one has also to calculate expression (18) first.

\section{A SPIN-1 BOSE GAS}

In this section we illustrate the feasibility of the proposed approach on a set of spin-1 bosons confined in a harmonic potential, based on the partition function (18) of the mixture. For simplicity, the many-body interaction, studied in [1,2] to allow for oscillations of the center of mass with a different frequency than that of the oscillations of the internal degrees of freedom, is not taken into in consideration here. This leads to a simpler albeit non trivial model. The calculation techniques can almost entirely be based on those that have been used for the polarized case [1]:2].

The generating function $\Xi(\beta, u)=\sum_{N_{m}=0}^{\infty} \mathbb{Z}\left(\beta \mid N_{m}\right) u^{N_{m}}$ of the partition function $\mathbb{Z}\left(\beta \mid N_{m}\right)$ of each component in the mixture is known from these previous calculations, and given by:

$$
\Xi(\beta, u)=\exp \left(\sum_{\ell=1}^{\infty} \frac{1}{\ell} \frac{b^{\frac{3}{2} \ell} u^{\ell}}{\left(1-b^{\ell}\right)^{3}}\right) \text { with } b=e^{-\beta \Omega_{m}} .
$$

It should be noted that $u$ and $b$ depend on the parameters of the spin-polarized subsystem. In the actual calculation we consider the same confining frequencies $\Omega_{m}=w$ for each subsystem (not exploiting the possibility of different confining potentials for different spin states).

There exist many ways to derive recursion relations between the partition functions with

a different number of particles [40 44]. A binomial expansion of the generating function almost immediately leads to:

$$
\mathbb{Z}(\beta \mid N)=\frac{1}{N} \sum_{n=0}^{N-1}\left(\frac{b^{(N-n) / 2}}{1-b^{N-n}}\right)^{3} \mathbb{Z}(\beta \mid n) .
$$


Combining the solutions for $\mathbb{Z}(\beta \mid N)$ from the recursion relations, the partition function of the mixture becomes:

$\mathfrak{Z}_{\text {mixt }}(\beta \mid N)=\sum_{N_{-1}} \sum_{N_{0}=0}^{N_{-} N_{-1}} \frac{N ! \exp \left(\left(2 N_{-1}-N+N_{0}\right) \beta \omega_{s}\right)}{N_{-1} ! N_{0} !\left(N-N_{0}-N_{-1}\right) !} \mathbb{Z}\left(\beta \mid N_{-1}\right) \mathbb{Z}\left(\beta \mid N_{0}\right) \mathbb{Z}\left(\beta \mid N-N_{0}-N_{-1}\right)$.

which is the key result of the present paper. Once this function is known the thermodynamical quantities can be obtained in a straightforward manner.

\section{A. Specific heat and magnetic susceptibility}

The numerical representation of the results has to cope with very large numbers. For accuracy reasons the following transformation proves to be useful:

$$
\mathfrak{Z}_{\text {mixt }}(\beta \mid N)=N ! e^{N \beta \omega_{s}} b^{\frac{3}{2} N} S_{\text {mixt }},
$$

with

$$
S_{m i x t}=\sum_{m=0}^{N} \sum_{n=0}^{N-m} \exp \left(-\beta \omega_{s}(m+2 n)\right) \frac{\chi_{N-m-n} \chi_{m} \chi_{n}}{(N-m-n) ! m ! n !}
$$

where the scaling of the polarized partition function is given by:

$$
\mathbb{Z}(\beta \mid N)=b^{\frac{3}{2} N} \chi_{N}
$$

with the appropriate recursion relation:

$$
\chi_{N}=\frac{1}{N} \sum_{m=0}^{N-1} \frac{\chi_{m}}{\left(1-b^{N-m}\right)^{3}}, \text { with } \chi_{0}=1
$$

Remembering the definition $\omega_{m}=\mu m|\mathbf{B}|$, it is clear that $\omega_{s}$ denotes the maximal possible frequency due to the magnetic field.

The expected number of particles $\left\langle N_{m}(\beta \mid N)\right\rangle$ in each spin state $m$ then takes the following form suitable for numerical computation: 


$$
\begin{aligned}
\left\langle N_{1}(\beta \mid N)\right\rangle & =\frac{1}{S_{m i x t}} \sum_{m=0}^{N} \sum_{n=0}^{N-m} n \exp \left(-\beta \omega_{s}(m+2 n)\right) \frac{\chi_{N-m-n} \chi_{m} \chi_{n}}{(N-m-n) ! m ! n !} \\
\left\langle N_{0}(\beta \mid N)\right\rangle & =\frac{1}{S_{m i x t}} \sum_{m=0}^{N} \sum_{n=0}^{N-m} m \exp \left(-\beta \omega_{s}(m+2 n)\right) \frac{\chi_{N-m-n} \chi_{m} \chi_{n}}{(N-m-n) ! m ! n !} \\
\left\langle N_{-1}(\beta \mid N)\right\rangle & =N-\left\langle N_{0}(\beta \mid N)\right\rangle-\left\langle N_{1}(\beta \mid N)\right\rangle .
\end{aligned}
$$

The same parameterization can be used to obtain the internal energy of the mixture

$$
U_{\text {mixt }}\left(\beta \mid N, \omega_{s}\right)=-\frac{d}{d \beta} \ln \left(\mathfrak{Z}_{\text {mixt }}(\beta \mid N)\right)
$$

leading to

$$
\begin{aligned}
U_{\text {mixt }}\left(\beta \mid N, \omega_{s}\right)=N\left(\frac{3}{2} w-\omega_{s}\right)+\frac{1}{S_{\text {mixt }}} \sum_{m=0}^{N} \sum_{n=0}^{N-m} e^{-\beta \omega_{s}(m+2 n)} \frac{\chi_{N-m-n}}{(N-m-n) !} \frac{\chi_{m}}{m !} \frac{\chi_{n}}{n !} \\
\times\left(U_{N-m-n}+U_{m}+U_{n}+\omega_{s}(m+2 n)\right)
\end{aligned}
$$

(with $U_{m}$ the internal energy of the particles in spin state $m$ ). The specific heat

$$
C\left(\beta \mid N, \omega_{s}\right)=\frac{d}{d T} U_{\text {mixt }}\left(\beta \mid N, \omega_{s}\right)
$$

and the magnetic susceptibility $\frac{d}{d B} U_{\text {mixt }}(\beta \mid N)$, proportional to $\frac{d U\left(\beta \mid N, \omega_{s}\right)}{d \omega_{s}}$, are easily obtained from this expression.

In fig. 1 we show the specific heat, the magnetic excess specific heat (i.e. the contribution to the specific heat due to the magnetic field) and the magnetic susceptibility for 100 bosons. For 1000 bosons the same quantities are shown in fig 2. The temperature is expressed in units of $T_{c}=\frac{w}{k}\left(\frac{N}{\zeta(3)}\right)^{1 / 3}$, where $w$ is the frequency parameter of the confinement, $k$ is the Boltzmann constant and $\zeta(3)=1.202056903$ is a Riemann zeta-function. The frequency parameter for the internal degrees of freedom is expressed in units of $w$ :

$$
\omega_{s}=w_{s} w
$$

Using these units the expression for $b$ becomes $b=\exp \left(-\frac{1}{t}\left(\frac{\zeta(3)}{N}\right)^{1 / 3}\right)$ with $t=\frac{T}{T_{c}}$ and $\exp \left(-\beta \omega_{s}\right)=b^{w_{s}}$. Because there is a substantial dependence on the magnetic field strength, the influence of the magnetic field can be illustrated by the redistribution of the particles over 
the internal degrees of freedom, giving rise to the magnetic excess specific heat. The magnetic susceptibility clearly illustrates the dependence of the internal energy on the magnetic field strength.

\section{B. The Schottky anomaly}

In the specific heat plotted in fig. 1 and fig. 2 we have identified the low temperature maxima as Schottky anomalies. This contribution to the specific heat is attributed to the lifting of the degeneracy of the levels of the spin degrees of freedom, due to the magnetic field. The effect occurs irrespective of the boson statistics, as will be illustrated below.

For up to 6 particles we calculated all polarized partition functions by symbolic algebra. This allows to study the free energy and the derived quantities exactly. Because the same calculation can be performed using Maxwell-Boltzmann statistics, we may isolate the effect of the Bose-Einstein statistics on the Schottky anomaly.

For numerically exact calculations it is useful to factorize out the dominant behavior

$$
\mathbb{Z}(\beta \mid N)=z_{N}(\beta) \frac{b^{\frac{3 N}{2}}}{\prod_{n=1}^{N}\left(1-b^{n}\right)^{3}} .
$$

The recursion relation for the resulting polynomials $z_{N}(\beta)$ becomes then:

$$
z_{N}(\beta)=\frac{1}{N} \sum_{n=0}^{N-1} z_{n}(\beta) \frac{\prod_{j=n+1}^{N}\left(1-b^{j}\right)^{3}}{\left(1-b^{N-n}\right)^{3}} .
$$

The polynomials $z_{N}(\beta)$ for $N=1, \ldots, 6$ were obtained by integer arithmetic and the results of this calculation are listed in table 1 . For low temperatures $(k T \ll w / 2)$ the specific heat is plotted in fig. 3 as a function of the temperature and of the magnetic field. The contribution associated with the lifting of the degeneracy and the evolution to a single polarized state with increasing magnetic field clearly manifests itself. We have also studied the specific heat of 6 distinguishable particles with spin degrees of freedom. The result is shown in fig. 4, and it also exhibits a maximum due to the Schottky anomaly. Comparing the specific heat of distinguishable particles with that of bosons, it should be noted that the 
Bose-Einstein statistics only weakly influences the magnitude of Schottky anomaly. But its relative contribution is more pronounced for Bose-Einstein statistics than it is for MaxwellBoltzmann statistics because the boson character suppresses the energy fluctuations at low temperature leading to a smaller specific heat.

\section{DISCUSSION AND CONCLUSION}

First we review our method, then we comment on our results for the spin-1 model and finally we will conclude with a brief indication of the potential use of the method.

A first striking difference between the method worked out here and more standard approaches to the many-body problem is that we incorporate the statistics using directly the representation theory of the symmetric group instead of the more common second quantization. As we indicated before, Ginibre's approach is based on the grand canonical ensemble, whereas we based our method for particle-conserving systems on the distribution of states, which we transformed to the partition function and its generating function. The temperature and the chemical potential obtain their standard meaning from the saddle-point method used to invert the transform. The numerical analysis for the spin-polarized case published earlier [2,13] indicates that when there are sufficiently many particles, corrections to the saddle point inversion become negligible. We believe that this remains the case for unpolarized systems but we did not prove this yet.

When internal degrees of freedom are involved, we argued that a Feynman-Kac functional averaged over an indexed Brownian motion describes the evolution of the system. The projection on the appropriate irreducible representation of the permutation group leads to a multinomial combination of the spin states in the partition function. The index process to distinguish between different spin states is not used explicitly, because only the diagonal part of the spectral representation of the propagator of that process is needed for the thermodynamics. The fact that we can construct a process for the evolution of the internal

degrees of freedom is nevertheless important, not only for a better understanding of the 
behavior of the system at long Euclidean times (low temperature) but also for considering correlations between components in different internal states. We indicated briefly that two-body interactions can be taken into account by a cumulant expansion of the exponential in the Feynman-Kac functional. This implies that one-point, two-point and $n$-point correlation functions have to be calculated. Other expansions, like e.g. the Mayer cluster expansion, can presumably also be carried out in our formalism, but this point deserves further investigation.

In order to demonstrate the feasibility of the approach, we gave an example based on an exactly soluble harmonically confined spin-1 boson gas in a magnetic field and calculated the internal energy, the magnetic susceptibility and the specific heat for the system in equilibrium. Furthermore, although we illustrated the technique without two-body interactions, it should be noted that the variational method that we have applied to the spin-polarized case can also be used to study mixtures of identical particles with different degrees of freedom.

The example itself, a noninteracting boson gas, exhibits Bose-Einstein condensation characterized by one of the maxima in the specific heat as a function of temperature. A second maximum is due to the Schottky contribution to the specific heat. It should be noted that the Schottky anomaly indicates that the $z$-component of the total spin, which is zero in the ground state, acquires values different from zero. The fact that this occurs at a lower temperature than the BEC for an ideal system may of experimental importance.

We conclude that the methods which we developed for polarized systems of identical particles can also be extended to non-polarized systems, as shown in the present paper. The basic tools are a Feynman-Kac functional with an adapted process, the projection on the symmetric or anti-symmetric representations, and the path integral evaluation of the averages. 


\section{ACKNOWLEDGMENTS}

This work is performed within the framework of the FWO projects No. 1.5.729.94, 1.5.545.98, G.0287.95, G.0071.98 and WO.073.94N (Wetenschappelijke Onderzoeksgemeen-

schap over "Laagdimensionele systemen"), the "Interuniversitaire Attractiepolen - Belgische Staat, Diensten van de Eerste Minister - Wetenschappelijke, Technische en Culturele Aangelegenheden", and in the framework of the BOF NOI 1997 projects of the Universiteit Antwerpen.

[1] F. Brosens, J. T. Devreese, and L. F. Lemmens, Phys. Rev. E 55, 227 (1997); E 55, 6795 (1997).

[2] F. Brosens, J. T. Devreese, and L. F. Lemmens, Phys. Rev. E 57, 3871 (1998); E 58, 1634 (1998).

[3] J. Ginibre, J. Math. Phys. 6, 238 (1965); 6, 252 (1965); 6, 1432 (1965).

[4] J. Ginibre in Statistical Mechanics and Quantum Field Theory, 1971, Les Houches Lectures, edited by C. de Witt and R. Stora (Gordon and Breach, New York, 1971).

[5] A. P. Lyubartsev and P. Vorontsov-Velyaminov, Phys. Rev. A 48, 4075 (1993).

[6] P. N. Vorontsov-Velyaminov, S. D. Ivanov, and R. I. Gorbunov, Phys. Rev. E 59, 168 (1999).

[7] F. Cornu, Phys. Rev. E 53, 4562 (1996).

[8] A. Alastuey and A. Perez, Phys. Rev. E 53, 5714 (1997).

[9] F. Cornu, Phys. Rev. E 58, 5268 (1998); 5293 (1998); 5322 (1998).

[10] D. C. Brydges and Ph. A. Martin, Coulomb systems at low density, cond-mat/9904122

[11] R. P. Feynman, Statistical Mechanics, a Set of Lectures, W. A. Benjamin Inc., Reading, 1972. 
[12] R. Kubo, Statistical Mechanics, North-Holland, Amsterdam, 1974.

[13] L. F. Lemmens, F. Brosens, and J. T. Devreese, Solid State Commun. 109, 615 (1999); condmat/9803168.

[14] P. Borrmann, J. Harting, O. Muelken, and E. Hilf, Phys.Rev. A 60, 1519 (1999).

[15] H. Sato, Phys. Rev. C 36, 785 (1987).

[16] N. F. Johnson and M. C. Payne, Phys. Rev. Lett. 67, 1157 (1991); Phys. Rev. B 45, 3819 (1992).

[17] A. Angelucci and A. Tagliacozzo, Phys. Rev. B 50, 7088 (1997).

[18] D. Lynden-Bell, R.M. Lynden-Bell, Proc.Roy. Soc. A (London) 455, 475 (1999); condmat/9808173

[19] D. Lynden-Bell, R.M. Lynden-Bell, cond-mat/9904019

[20] Chandrasekhar, Newton's Principia for the Common Reader, Oxford U.P., Oxford 1995.

[21] I. Newton, Principia, problem 64, Royal Society London 1687.

[22] L. F. Lemmens, F. Brosens and J. T. Devreese, Phys. Rev. A 59, 3112 (1999); e-print condmat/9901102.

[23] P. Gruter, D. Ceperley, and F. Laloë, Phys. Rev. Lett. 79, 3549 (1997).

[24] J. Tempere, F. Brosens, L. F. Lemmens, and J. T. Devreese, Solid State Commun. 107, 51 (1998).

[25] L.F. Lemmens Phys Lett. A 222 , 419 (1996).

[26] S. Foulon, F. Brosens, J. T. Devreese, and L. F. Lemmens, Phys. Rev. E 59, 3911 (1999).

[27] M. H. Anderson, J. R. Ensher, M. R. Matthews, C. E. Wieman, and E. A. Cornell, Science 269, 198 (1995). 
[28] K. B. Davis, M.-O. Mewes, M. R. Andrews, N. J. Van Druten, D. S. Durfee, D. M. Kurn, and W. Ketterle, Phys. Rev. Lett. 75 , 3969 (1995).

[29] C. C. Bradley, C. A. Sackett, J. J. Tollett, and R. G. Hulet, Phys. Rev. Lett. 75, 1687 (1995).

[30] C.C. Bradley, C.A. Sacket, and R.G. Hulet, Phys. Rev. Lett. 78, 985 (1997).

[31] R. Rossignoli, Phys. Rev. C 51, 1772 (1995).

[32] T.-L.Ho and V. B. Shenoy, Phys. Rev. Lett. 77, 3276, (1996).

[33] H.-J. Miesner, D.M. Stamper-Kurn, J. Stenger, S. Inouye, A. P. Chikkatur, and W. Ketterle, Phys. Rev. Lett.82, 2228 (1999).

[34] E. Timmermans, Phys. Rev. Lett.81, 5718 (1998).

[35] L. F. Lemmens, F. Brosens and J. T. Devreese, Physica B, proceedings LT22 (to be published).

[36] B. Simon, Functional Integration and Quantum Physics, Academic Press, New York, 1979.

[37] G. Roepstorff, Path Integral Approach to Quantum Mechanics, Springer, Berlin, 1994.

[38] L. S. Schulman, Techniques and Applications of Path Integration, J. Wiley \& Sons, New York, 1981.

[39] H.-T. Elze and W. Greiner, Phys. Rev. A 33, 1879-1891 (1986)

[40] S. Grossman and M. Holthaus, Z. Naturforsch. 50a, 323; 921 (1995).

[41] S. Grossman and M. Holthaus, Phys. Lett. A 208, 188 (1995).

[42] K. Kirsten and D. J. Toms, Phys. Rev. A 54, 4188 (1996).

[43] W. Ketterle and N. J. van Druten, Phys. Rev. A 54, 656 (1996).

[44] P. Borrmann, G. Franke, J. Chem. Phys. 98, 2484 (1993). 


\section{Table}

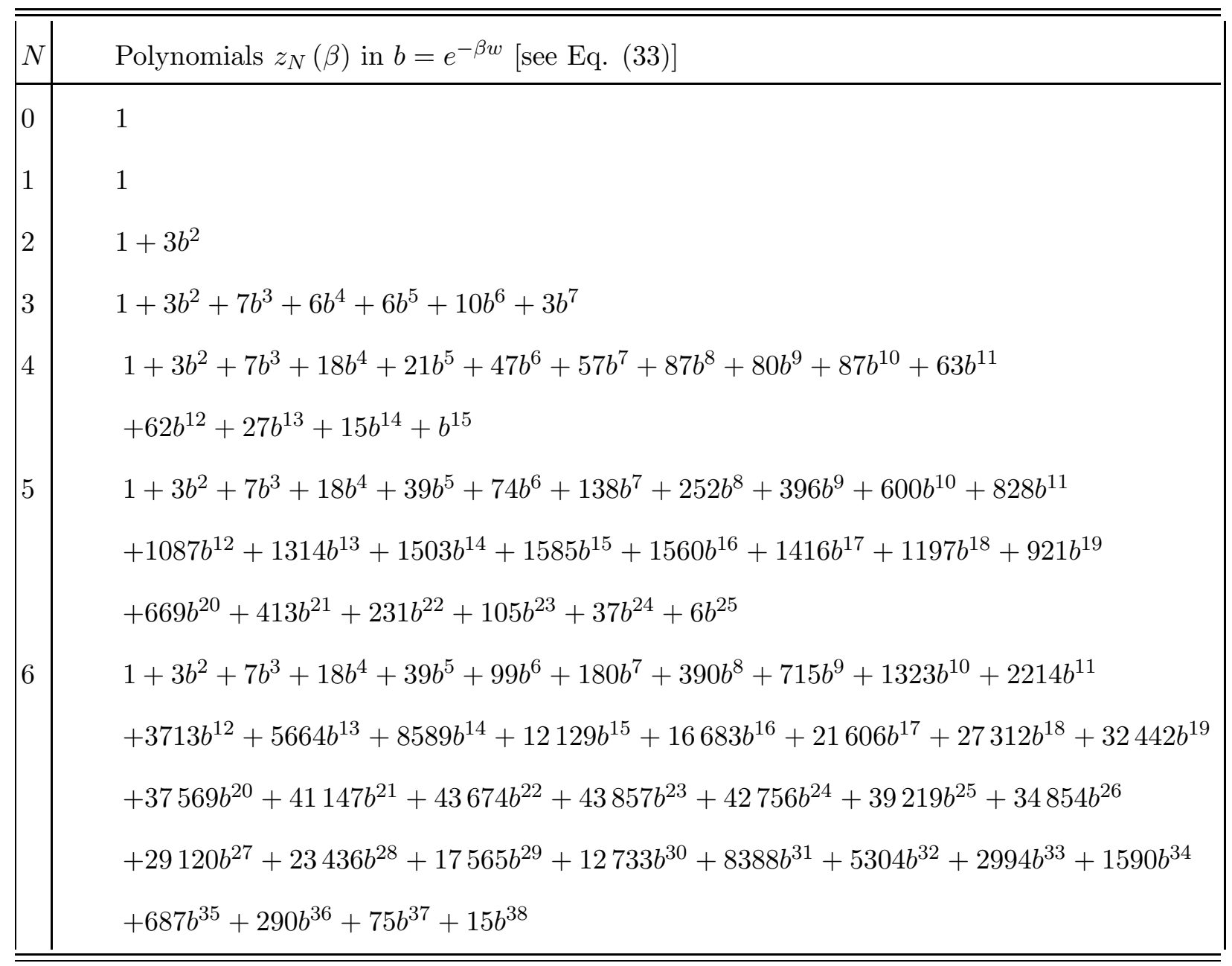

TABLE I. In this table we list the polynomials $z_{N}(\beta)$ for $N=1, \ldots, 6$. The coefficients are obtained using integer arithmetic and symbolic algebra. 


\section{Figure captions}

Fig. 1: For 100 bosons the specific heat per particle (a), the magnetic excess specific heat per particle (b), and the magnetic susceptibility (c) are shown as a function of the temperature and the magnetic field.

Fig. 2: For 1000 bosons the specific heat per particle (a), the magnetic excess specific heat per particle (b), and the magnetic susceptibility (c) are shown on the same scale as in fig 1 .

Fig. 3: The specific heat per particle is shown for 6 bosons distributed over 3 spin states in thermal equilibrium. The maximum in the $\left(C, w_{s}\right)$-plane with fixed $T$ is identified as the Schottky anomaly. This is an entropic effect due to the lifting of the degeneracy of the spin degrees of freedom by an external field.

Fig. 4: The specific heat per particle for 6 distinguishable particles is shown with MaxwellBoltzmann statistics. Note the presence of the Schottky anomaly. This figure should be compared with figure 3 where the same quantity is shown taking the Bose Einstein statistics into account. 


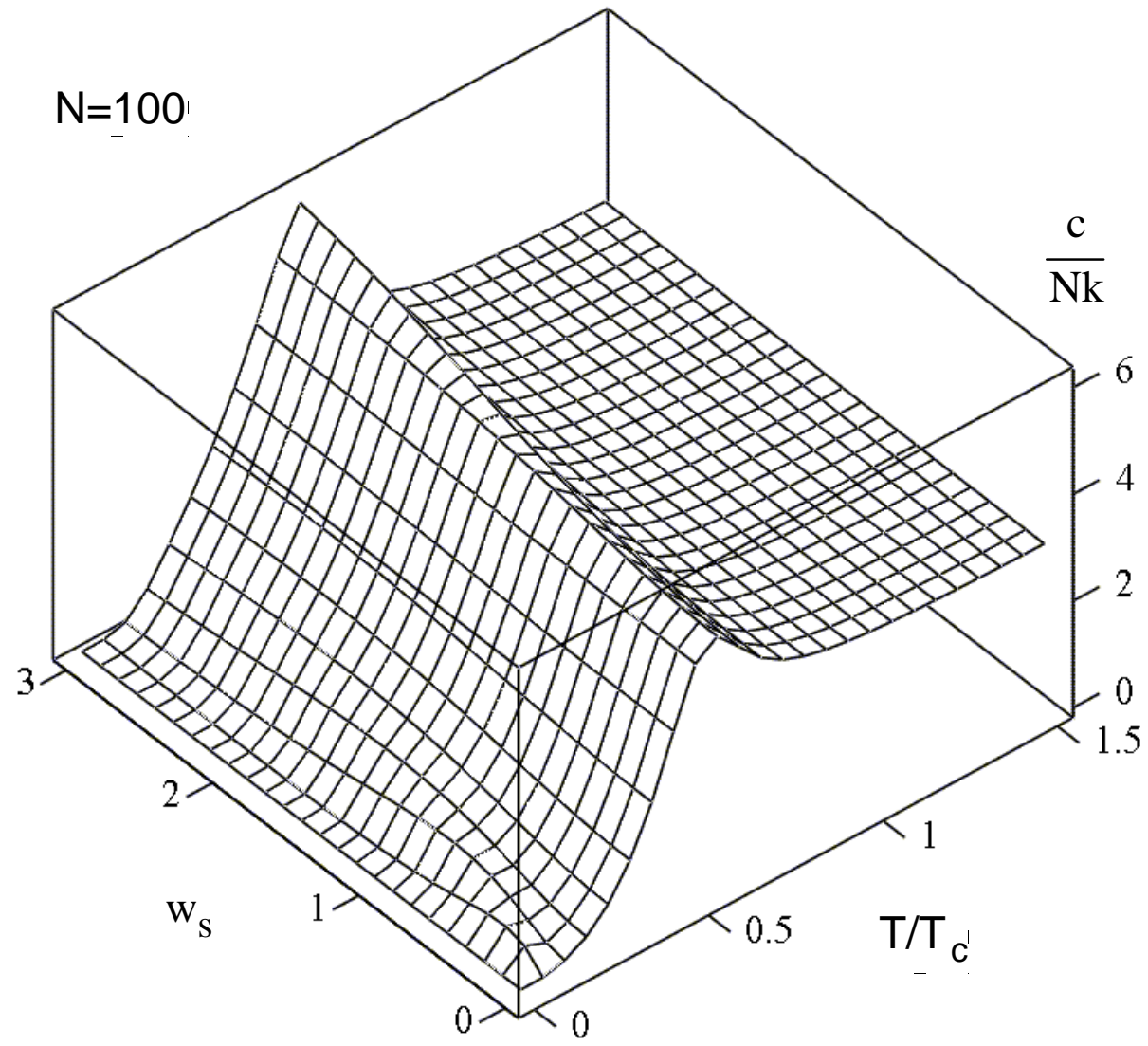

Figure 1 (a) 


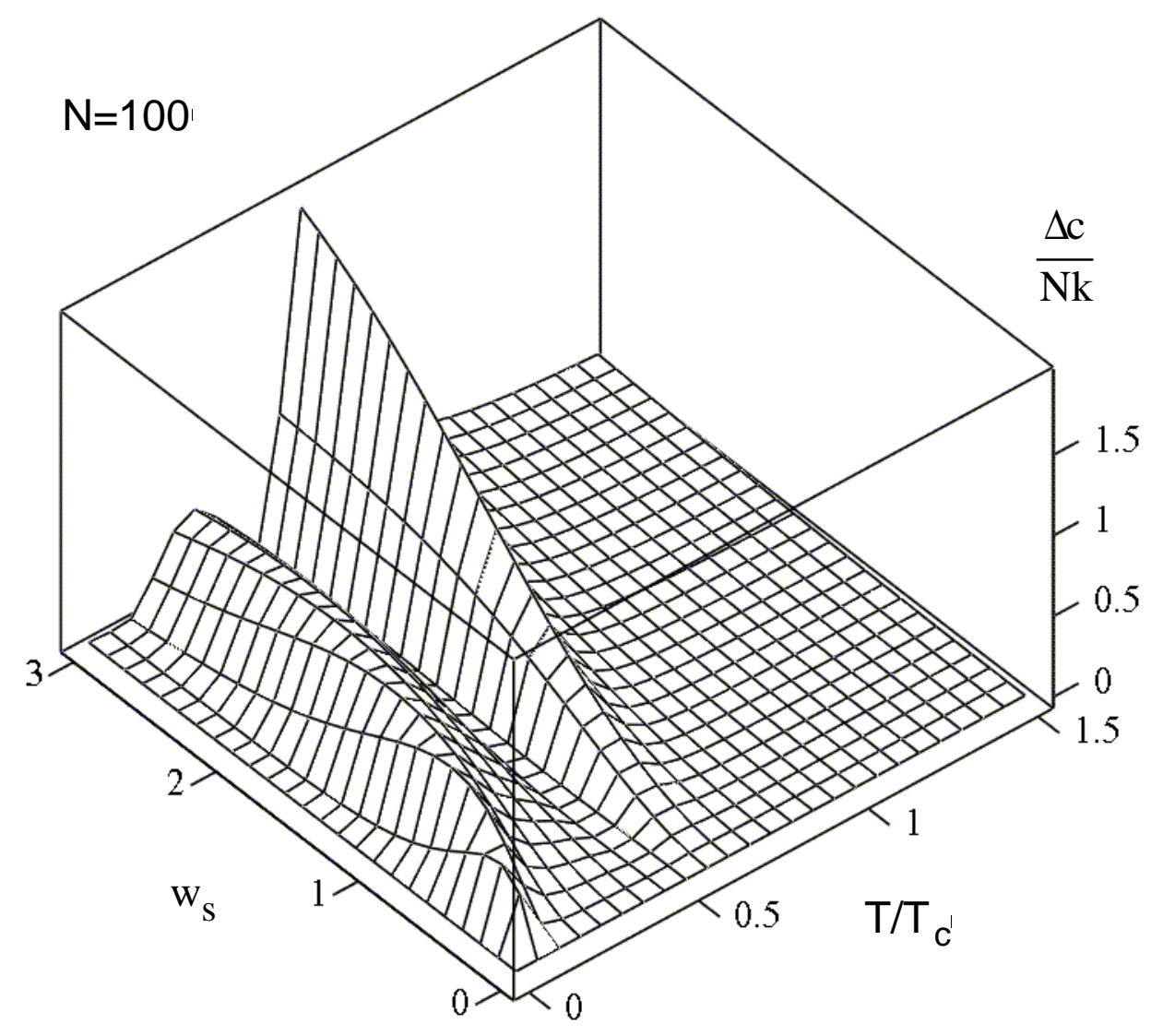

Figure 1 (b) 


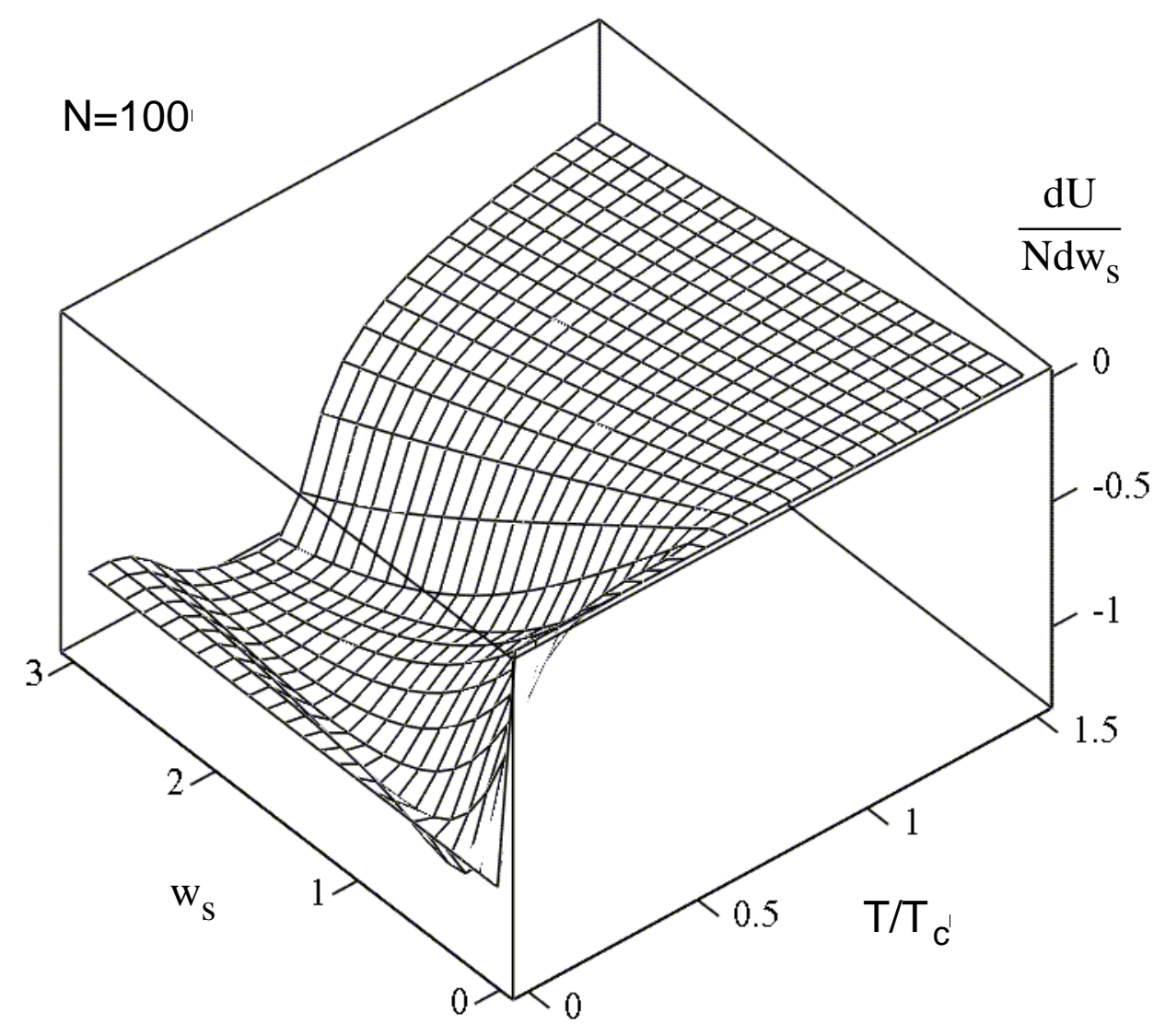

Figure 1 (c) 


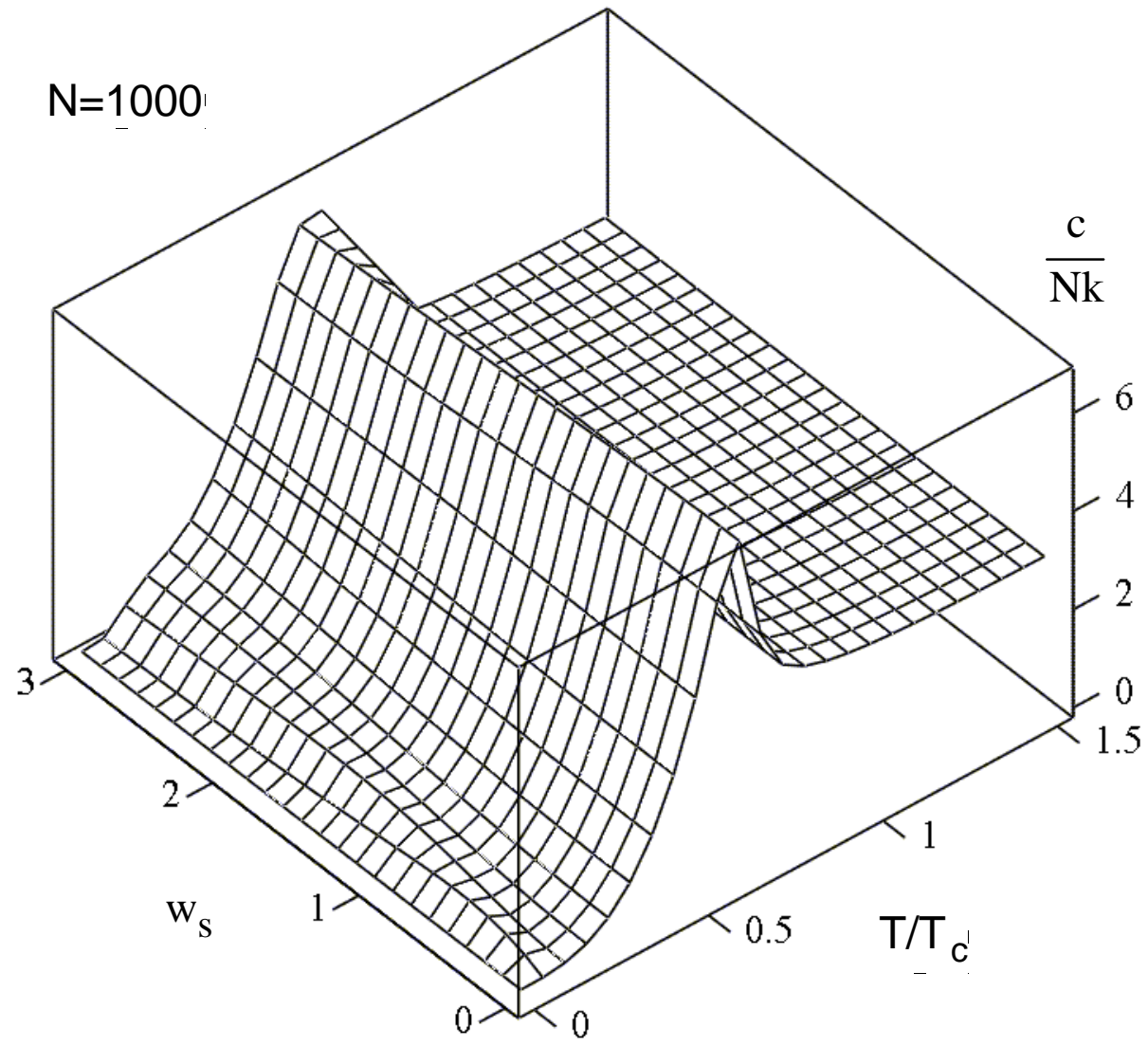

Figure 2 (a) 


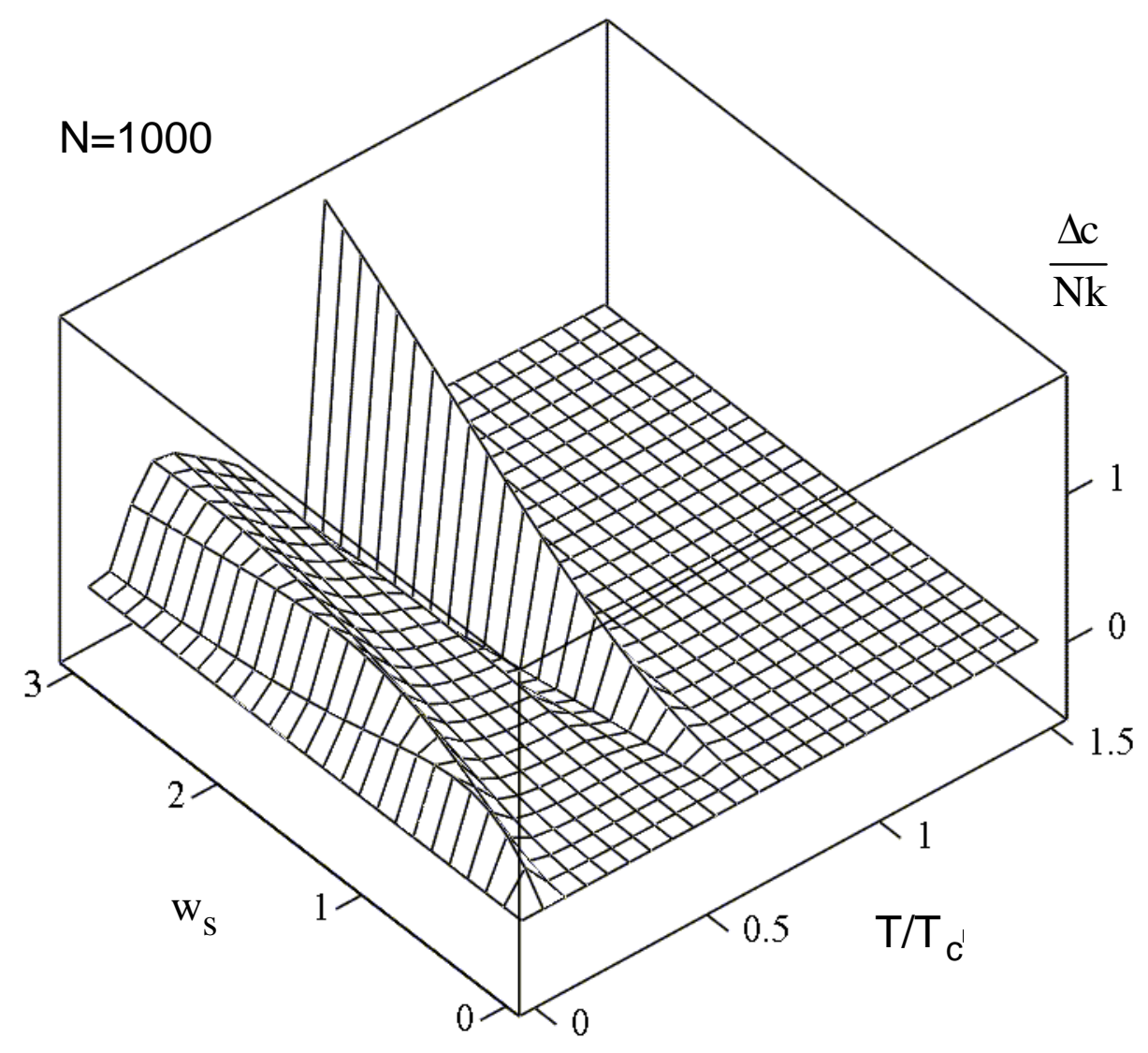

Figure 2 (b) 


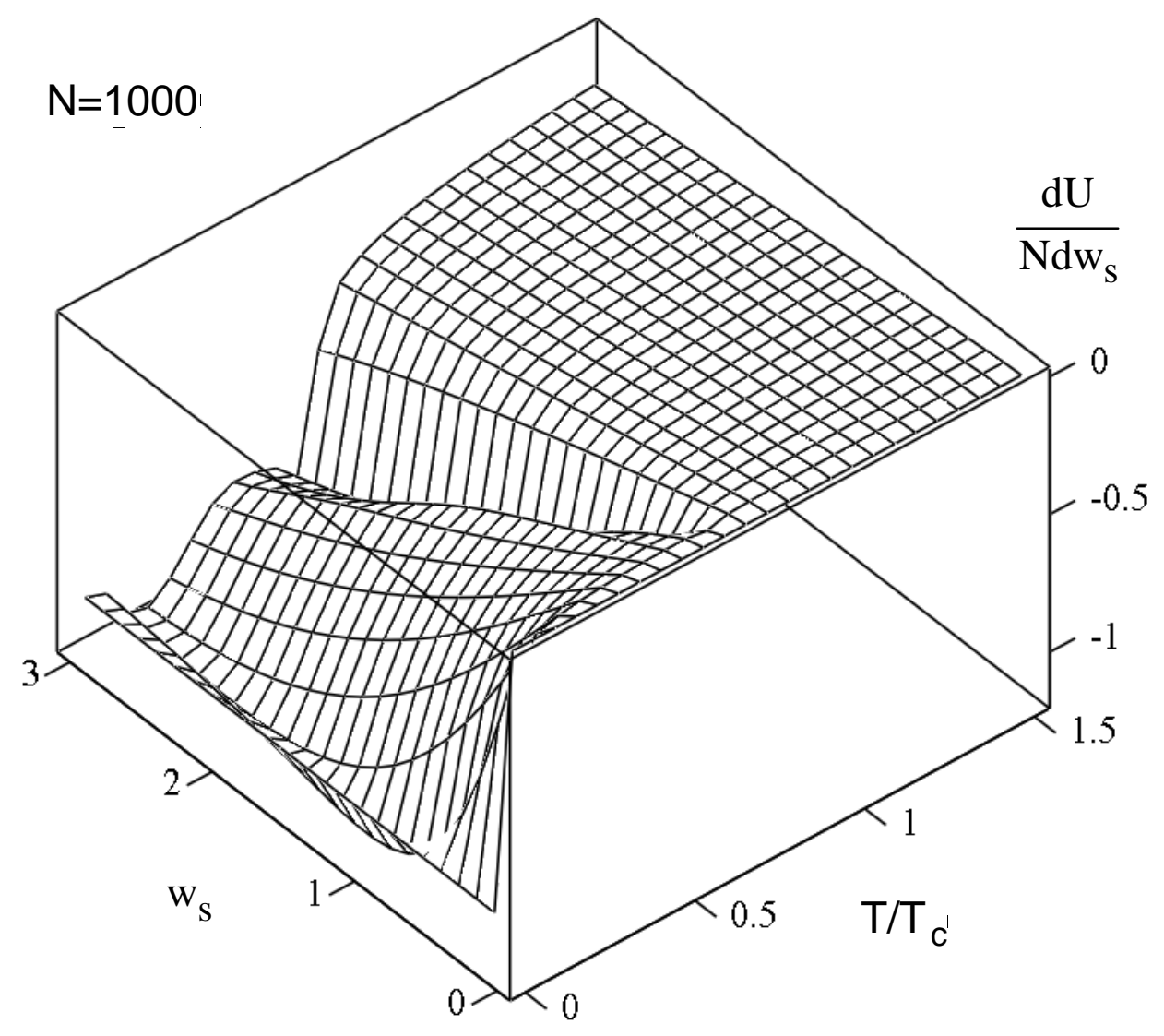

Figure 2 (c) 


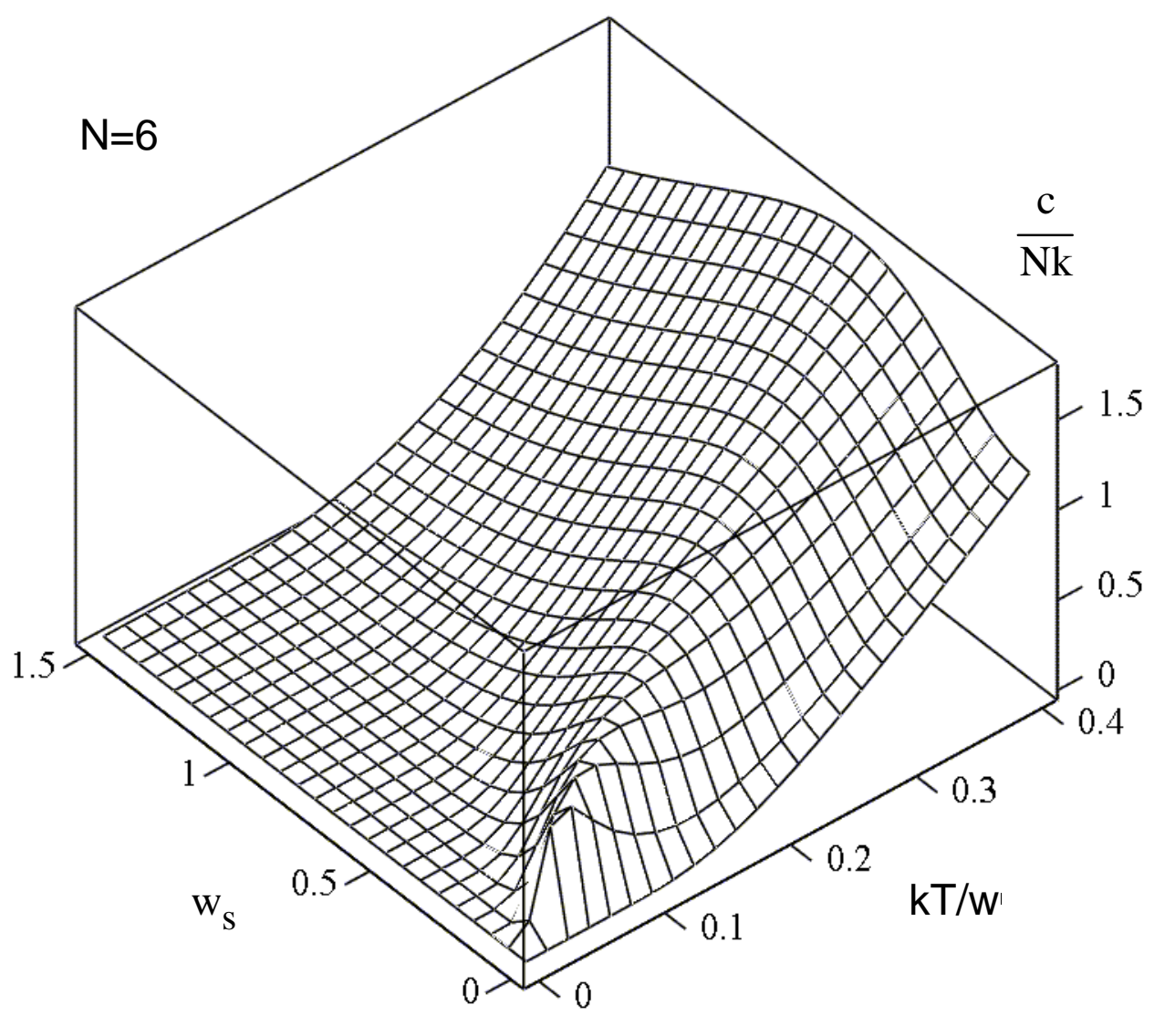

Figure 3 


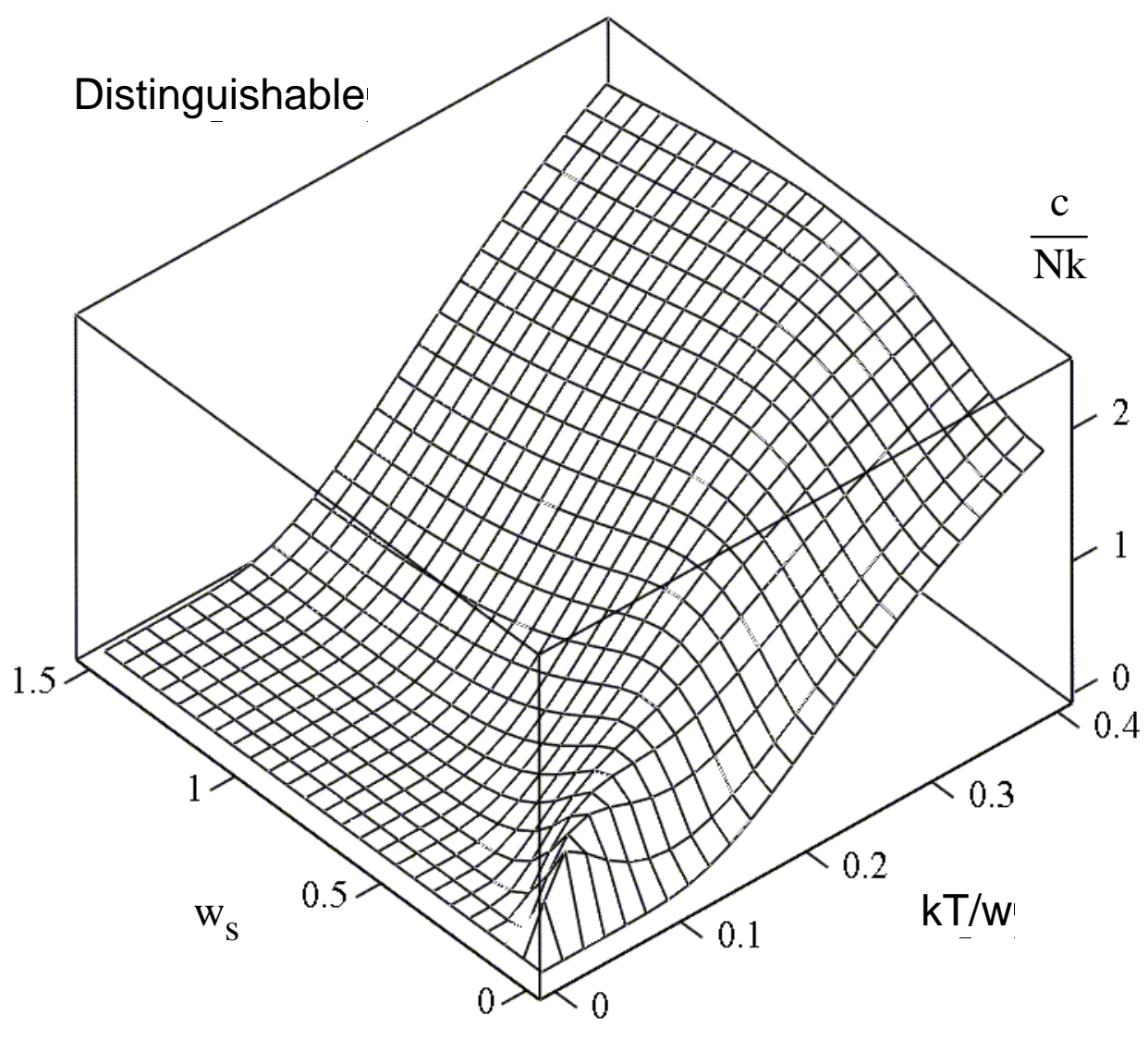

Figure 4 